\title{
Visiones Dominantes de la Política Educacional en la Transición Chilena hacia la Democracia (1990 - 2010)
}

\author{
Visões dominantes da política educacional na transição chilena \\ à democracia (1990-2010)
}

\section{Dominant views of educational policy in the Chilean transition to democracy $(1990-2010)$}

\section{Sebastián Donoso-Díaz Moyra Castro-Paredes Giselle Davis -Toledo'}

\section{Resumo:}

O texto descreve em forma suscinta os principais componentes das visões dominantes da política educacional chilena no período recenté de transição pós-ditadura, que vai do ano de 1990 até começos de 2010, coincidindo com os quatro governos que em forma sucessiva a coalização política denominada "concertación de Partidos por la Democracia" ganhou as eleições presidenciais, até que em março de 2010 assumiu a coalizão de direita que governará até março de 2014. A revisão dos enfoques da política educacional é relevante para compreender os sucesos ocorridos, como também dá conta de algumas determinantes que incidem nas propostas de políticas hoje em curso, permitindo delinear os desafíos que se avizinham. Em essência, aponta que esses vinte anos se caracterizaram pelas tensões e conflitos entre uma visão de estado social e uma neoliberal, a que não se resolve senão até a segunda década analisada, com o predominio da visão neoliberal.

Palavras-chave: Política educacional; Chile; Transição democrática; Visões contrapostas de sociedade.

\section{Resumen:}

El texto describe en forma sucinta los principales componentes de las visones dominantes de la política educacional chilena en el reciente período de la transición postdictadura, que va del año 1990 hasta comienzos del 2010, coincidiendo con los cuatro gobiernos que en forma sucesiva la coalición política denominada "concertación de Partidos por la Democracia" ganó las elecciones presidenciales, hasta que en marzo del año 2010 asume la coalición de derecha que gobernará hasta marzo del 2014. La revisión de los enfoques de la política educacional es relevante para comprender los sucesos ocurridos, como a su vez da cuenta de algunas determinantes que inciden en las propuestas de políticas hoy en curso, permitiendo delinear los desafíos que se avecinan. En lo esencial plantea que estos veinte años se caracterizaron por las tensiones y conflictos entre 
una visión de estado social y una neoliberal, la que no se resuelve sino hacia la segunda década en análisis, con el predominio de la visión neoliberal.

Palabras claves: Política educacional; Chile; Transición democrática; Visones contrapuestas de la sociedad.

\begin{abstract}
:
The text succinctly describes the main components of the dominant visions of Chilean education policy in the recent post-dictatorship transition period, which runs from 1990 to early 2010, coinciding with the four governments in succession form coalition siva policy called "coalition of Parties for Democracy" won the presidential election, until March of 2010 assumes the right-wing coalition that will rule until March 2014. The review of approaches to education policy is relevant to understanding the cases occurred successively, as in turn realizes some determinants that influence the policy proposals now under way, allowing outline the challenges ahead. Essentially argues that these twenty years were characterized by tensions and conflicts between social vision and a neoliberal state, which is not solved but by the second decade of analysis, with the predominance of neoliberal vision.
\end{abstract}

Keywords: Educational policy; Chile; Democratic transition; Conflicting visions of society.

\title{
1. Presentación
}

El sentido del texto es exponer sucintamente las visiones dominantes en la política educacional chilena en las dos décadas en análisis (1990 -2010), que conforman un período de tiempo de gran relevancia para el sector ya que corresponden a época de recuperación democrática, marcada por un conjunto de propuestas, iniciativas y programas que se llevaron a cabo, en un contexto sociopolítico que da píe a la hipótesis de trabajo que asume que los principales conflictos experimentados en le período en cuestión por el sector educacional, tienen su origen en la coexistencia de dos lógicas de operación en su interior, claramente diferenciables, cuya dinámica se funda en orientarse a distintos modelos de organización de la sociedad. Materia que ha estado marcada además -operativamente- por la incapacidad inicial de dominio de una visión sobre la otra. Este fenómeno, con el transcurrir del tiempo hacia fines de la segunda década en análisis (2010), se va definiendo finalmente hacia la lógica de mercado por sobre la perspectiva de la educación como bien público, enfoque que corresponde a una segunda hipótesis que se sostiene en el texto².

Estas visiones contrapuestas reconocen en su esencia a concepciones alternativas del Estado y de la sociedad, y por lo tanto al diseño e implementación de políticas que generan conflictos y tensiones complejas de equilibrar, lo que se percibe en la forma como en el campo en análisis se han "articulado y resuelto" las políticas educacionales en esos años tan significativos.

El período de años en cuestión (1990 a 2010), corresponde a una etapa política clave del desarrollo del sistema social y educacional chileno, marcado por lo que se denomina la transición política hacia la democracia (Garretón y Garretón, 2010), cuyos hitos en este plano son definidos como un ejercicio de continuidad que proveyó la coalición política que gobernó en las dos décadas en estudio: la Concertación de partidos por la Democracia, el cual -por cierto- es un escenario muy diferente al de las casi dieciocho años de gobierno dictatorial que le anteceden, en el cual el Estado redefinió su rol y su actuar, siguiendo una concepción subsidiaria algo particular que se implementa en Chile (Eaton, 2004), y que finalmente logra imponerse y se mantiene también en la era democrática de la que damos cuenta, entre otros motivos gracias una fuerte estructura normativa en educación que logra ser removida parcialmente en el año 2008, al sustituirse la Ley Orgánica Constitucional de Enseñanza (LOCE), instalada en el

2 Algunas perspectivas señalan que siempre el neoliberalismo dominó en esta materia, y que solamente en algunos hitos puntuales se impuso una visión más pro-derecho educativos en su sentido amplio. No obstante lo señalado por algunos, los autores del texto creemos que hubo una disputa en la visión de la educación que se mantuvo con fuerza en la primera década y fue perdiendo espacio desde mediados de la segunda etapa de los gobiernos concertacionistas. 
ocaso dictatorial, cuyo finalidad era instalar una "camisa de fuerza" al hacer del Estado en educación, y que como conciencia del movimiento estudiantil del año 2006, es finalmente reemplazada por una Ley que denota avance, aunque su tramitación es fruto de concesiones hacia la derecha conservadora (Bellei, et al. 2009).

Dos argumentos directamente relacionados fundamentan la elección del periodo de años en estudio: primero, lo recientemente expuesto acerca del rol de la Concertación de Partidos por la Democracia como un hito histórico estratégico para el desarrollo del país, y en segundo lugar, el análisis de los conflictos, tensiones y equilibrios entre los modelos político económicos de sociedad que condicionan las tendencias de las políticas y en este caso particular de las políticas educativas, y que son determinantes respecto de su quehacer. Este protagonismo del sector educación se debe a que en forma creciente ésta va siendo un elemento capital de las demandas sociales, como también su diseño estructural le sitúa, precisamente, bajo un rol insustituible del modelo de desarrollo societal, sea por su papel en la construcción y validación del pacto social que se busca imperiosamente promover y sustentar, como por su función sobre la sustentabilidad del modelo económico que se busca adecuar a las nuevas condiciones sociales que surgen con la democracia (Donoso y Schmal, 2009).

\section{La valorización de "la educación" en la sociedad chilena}

Para la sociedad chilena en su conjunto la educación ha alcanzado una creciente importancia -en su versión de años de escolaridad- acorde a lo que ha ocurrido en otras latitudes. No obstante, en el caso en análisis Chile- mantienen este atributo, pese a ser uno de los cuerpos sociales con mayor desigualdad social del mundo, siendo la educación una meta relevante para sus ciudadanos y una conquista social de significación para la mayor parte de sus diferentes estratos sociales- De allí su fuerte gravitación en cotidianidad de lo público. Ello se refuerza dado que, a su vez, la educación es considerada un instrumento eficiente para el desarrollo social (movilidad) y económico (crecimiento), estando ambas funciones bien posicionadas de manera que son claves a la hora de respetar y proteger en todo pacto político que aspira al poder, y que dice relación no solamente con la dimensión escolar, sino también con la enseñanza superior y desde hace un tiempo con la preescolar.

En este marco de relevancia social de la educación, desde la perspectiva de la política educacional, las dos décadas en análisis constituyen una etapa relevante de estudio por una serie de características que se presentan en este período, cuya resolución fue compleja y con grados muy desiguales de logros, a saber: consolidar el proceso de universalización de la educación primaria y secundaria, incentivar al expansión del sistema de educación superior, responder a las crecientes demandas de democratización, aumentar el financiamiento, e instalar sistemas que estimulen y aseguren la calidad de la formación.

No obstante los avances registrados, el problema de mayor significación para el sistema escolar en su conjunto fue y sigue siendo, primero, que su calidad es claramente insuficiente y está por debajo de los requerimientos de aptitud que debiera alcanzar para contribuir en forma relevante al crecimiento y desarrollo social y económico del país, fenómeno ante el cual e s constantemente increpada por las entidades rectoras de esta materias -públicas y privadas- críticas que se han centrado más en la educación pública (Beyer, 2009), en las bases de la desigualdad social que generan Iso diferenciales de logros (Valenzuela et al., 2008).

En segunda instancia, hay evidencia que la calidad se distribuye desigualmente, de manera que los estratos de mayor capital social y económico, a su vez acceden a una educación de mayor calidad, fenómeno que no es nuevo, sino que históricamente sea presentado de esta manera, con bemoles, no obstante lo cual, los esfuerzos impulsados desde el Estado por cerrar las brechas en los veinte años considerados han sido variados e importantes, no obstante lo cual los resultados presentan un fuerte rezago, en cuanto al hecho que las brechas se reducen, pero a un ritmo muy lento, lejos de las expectativas y necesidades de una sociedad que necesita construir desde sus sistema educacional un pacto social inclusivo. Múltiples factores y condicionantes sociales contribuyen a generar y mantener esa situación, manifestándose en un desajuste periódico y "cíclico" 
del sistema educativo, seguido por intentos hasta ahora insuficientes para corregir los problemas de calidad (y consustancialmente de equidad) de la misma, así como también la profunda brecha entre las demandas sociales y las soluciones que ofrece la sociedad y el Estado, en especial en el sector educación.

La problemática señalada es de tal magnitud y profundidad que no puede ser asumida como una situación eventual que se corregirá por sí sola en tanto fenómeno natural permanezca como tal (desde la perspectiva de Hayek, 1982), sino por el contrario, más bien de no mediar transformaciones de relevancia en un plazo próximo de tiempo, tenderá a aumentar y profundizarse, afectando la gobernabilidad de la sociedad, tal cual o han hecho ver un serie de conflictos sociales que sean expresado recientemente entre los años 2010 al 2012 y que dicen don demandas de los diversos territorios por condiciones de desarrollo adecuadas, como ciertamente también se expresaron ele movimiento estudiantil del año 2011, que gozó de una gran base social.

Desde esta consideración resulta imperativo el análisis riguroso de los principales condicionantes sociopolíticos e hitos educativos que influyeron en las dinámicas de las políticas y reformas educativas impulsadas en estas décadas, con la finalidad de develar los mecanismos y lógicas que las rigieron, ya que de ello dependió su sustento y proyección en el tiempo, por cuanto, producto de la crisis educacional de los años 2006 y 2011, hay un pronóstico incierto sobre el desenlace inmediato de esta situación, cuestión no menor pues varias de las reivindicaciones estudiantiles, que gozaron de gran apoyo ciudadano, no han sido a la fecha debidamente resueltas, ni están en la agenda próxima de atención de los diversos organismos del estado, lo que acrecienta las posibilidades de revitalización de estos conflictos ${ }^{3}$.

\section{Las visiones de política educacional en tensión y conlficto}

Desde la perspectiva teórica, precisar el rol desempeñado por el Estado chileno y los principales protagonistas (actores) sociales y políticos, involucrados en el diseño e implementación de las políticas públicas en educación en el período de años considerados, por cuanto corresponden una etapa política clave para la gobernabilidad y gobernanza de la sociedad chile y de su instituciones públicas y privadas.

El análisis de la política educativa durante estas dos décadas de transición política puede considerarse atípico en el contexto socio democrático del país por los siguientes aspectos: (i) la continuidad de las políticas públicas y sociales, y en este marco de las educacionales por espacio de dos décadas, lo que muy pocas veces había sucedido en los doscientos años de vida independiente, sino por el contrario se trataba de ciclos breves, en muchas veces con sentido fundacional; (ii) en segunda instancia se impulsó a inicios del segundo Gobiernos concertacionista (Frei Ruiz- Tagle), una reforma educativa (1996 -2010) que primero es una de las de mayor data en América latina de las llamadas reformas de segunda generación; (iii) y porque su instalación se hace al unísono de las reformas en el continente, pero con una continuidad política mucho mayor; (iv) por que se instala en un país con un sistema de operación y financiamiento de la educación pública muy diferente de otros; esencialmente con sus sistema mixto con fuerte presencia de instrumentos de mercado (v) porque en la inspiración inicial de esta reforma educativa vuelve la creencia que el sector educación posee la fuerza para cambiar la sociedad (Braslavsky y Cosse, 1997; Gajardo, 1999), optimismo ya experimentado con las reformas de los 60', con el auge de la Teoría del Capital Humano (CEPAL/UNESCO, 1992), y (vi) porque en todo proceso de reforma, según Tedesco (1998), lo "innovador" reside en la forma cómo se priorizan, organizan e interactúan los componentes "de la política de reforma" esto es siguiendo a Bourdieu, en el campo.

3 Dos cuestiones capitales, intrínsecamente relacionadas no han sido atendidas ni de forma ni fondo por los organismos del Estado (Gobierno y Parlamento) medidas claras y directas de fortalecimiento de la educación pública, con implicancias ciertas en su organización y sistema de financiamiento, y en segundo lugar, todo lo vinculado con la gratuidad de la enseñan superiorlsde uns visiones de P superior.

4 Asumimos la política educativa como orientaciones que involucran el plano descriptivo- explicativo con el valorativo normativo. El ámbito de la política educativa está conformado por tres planos normativos: el jurídico, el crítico y el pedagógico (Fernández, 1999: 19). 
La interrelación entre determinantes sociales, dinámicas de los campos de acción social, visiones en juego y actores sociales dan lugar a la comprensión del racional que orientó las políticas educativas en las dos décadas en análisis de la política educativa en Chile (1990 - 2010).

Atendiendo a lo señalado, la interrelación entre los determinantes macro sociales como las micro sociales, -es decir aquellos contextos en que se sitúa (contextualiza) la acción educativa- se objetivan en lo que Bourdieu (1984,1991) denomina los campos de acción social, cuya noción de campo apela al escenario donde se produce y reproduce la acción social. Así, el campo es al mismo tiempo estructurado por los determinantes sociales, las redes sociales conformadas por las posiciones que ocupan los actores, dependiendo de los flujos de capital social, económico y simbólico que poseen y a los cuales acceden. La complejidad pedagógica, social y económica del fenómeno educativo requiere ser abordad desde su misma "complejidad", siendo el enfoque de Bourdieu una herramienta adecuada en tanto considera los determinantes macro sociales de época, las dinámicas de los contextos sociales y políticos específicos en los cuales se desarrolla e implementa la(s) política(s) educativa(s), así como también las visiones que orientan la acción social y las decisiones de sus principales protagonistas.

En este marco, las determinantes macro sociales son comprendidas como los paradigmas sociales y modelos económicos que modelan las tendencias políticas y demandas sociales en el ámbito educacional. Las dinámicas de los contextos sociales específicos en los cuales se generan las decisiones de política educativa, están determinadas por: (i) el conjunto de condiciones jurídico/ institucionales del sistema educativo chileno, (ii) la naturaleza misma de las problemáticas y las tensiones entre los poderes políticos en sus diversos ámbitos y niveles, y (iii) los estilos de gobernanza presentes en este escenario.

Si bien el concepto de campo implica determinación social, se considera también como una unidad dinamizada e indeterminada ya que los sujetos son considerados como actores sociales con poder de injerencia en la estructuración del mismo, lo que reduce tal determinación. Consecuentemente, es pertinente plantear la tercera dimensión consignada para el estudio, que remite a las visiones en conflicto y tensión al momento que los grupos de actores deciden, interpretan e implementan las políticas educativas.

El contexto socio histórico corresponde al campo de las políticas educativas, en tanto comprende la posición social de los grupos, los incidentes y tendencias de sus actores respectivos e incluye el orden existente que genera las políticas educativas. Por ello, en la impronta del desarrollo nacional, la educación ha sido estratégica en las propuestas de gobernabilidad de Aguirre Cerda, como para Frei Montalva y la Reforma Educacional de 1965 y para Allende Gossens y la ENU. También lo fueron para los cambios en el Gobierno Militar, con momentos cruciales, a saber: las Directivas Presidenciales para la Educación Chilena y la Reforma "silenciosa" de 1980, y en el ocaso de su gobierno la implantación de la Ley Orgánica de Enseñanza (LOCE), que evidencia la culminación de la relevancia que se le asignó a la Educación. Los sucesos posteriores a 1990 hasta el año 2010, son constitutivos del objeto de estudio y se enraízan en esa lógica.

Los primeros antecedentes del período en análisis se remiten a lo que denominamos la fase de pre-reforma educativa (1990-1995), en que las acciones emprendidas estuvieron marcadas por tres aspectos centrales: (i) desde 1982 el Gobierno venía reduciendo en forma sistemática y en grado importante el presupuesto del sector educación, al extremo que los recursos que éste asignó al primer año de ejercicio del Gobierno Democrático en 1990, representaron el 72\% del monto total actualizado del presupuesto del año 1982 (González, 2003, p. 610); (ii) la caída de los recursos financieros implicó un deterioro sostenido de la educación, esencialmente la escolar, cuyos impactos demoraron en revertirse más allá de la inflexión presupuestaria del año 1991; (iii) la reforma educativa de 1981 generó un problema 'invisible en el corto plazo' en la gestión financiera (Jofré, 1988) y en la pedagógica se asumió un modelo matricial sin puntos de intersección entre el Ministerio y los establecimientos escolares, corregido solamente y en parte en la Ley General de Educación de 2009.

En el marco país, el cambio del modelo de financiamiento de 1981 y la entrada en operación del enfoque de mercado implicó una alteración relevante en la composición de la matrícula escolar según dependencia escolar, 
introduciendo factores tensionantes en el sistema, vigentes hasta el momento. Antes de este proceso, el sector fiscal representaba el $80 \%$ de la matrícula total, el particular subvencionado el $15 \%$ y el privado un 5\%. Diez años después de la última cifra (1996), el sector municipal agrupaba el 56,5\% de la matrícula, el subvencionado el $33 \%$ y el particular el $8.7 \%$. Al año 2000, los cambios persisten pero cada vez con menor fuerza. Los municipales reúnen el 54,2\%, los subvencionados el 35,8\% y los privados el 8,2\% (Cox, 2003, p. 27):

El traspaso de los establecimientos fiscales a las municipalidades [ocurrido entre 1980 y 1986] replicó en muchos casos el centralismo a nivel comunal. Estas escuelas en la práctica no compiten entre sí por los alumnos. Están ausentes los incentivos para que ello ocurra. (BEYER et al. 2003, p. 187).

Las políticas educativas ${ }^{5}$ de los gobiernos de la transición democrática reaccionaron al deterioro de los resultados o al avance más lento de lo previsto, asumiendo dos lineamientos estratégicos: (i) el mejoramiento de los resultados educativos de la población, mediante intervenciones explícitas en el sistema educativo y, (ii) la actualización de sus competencias para su mejor incorporación al mercado de trabajo (Cox y González, 1997, p. 103). Al mismo tiempo que le dan continuidad al sistema de financiamiento escolar (subsidio) y formaliza el impulso a la Reforma Educativa de mediados de esa década. Esta continuidad ordena y da sustento a las acciones que en este campo se realizan desde los inicios de la transición, presentando una unidad de forma y fondo entre política y reforma educativa que reduce las tensiones en esta etapa al buscarse acuerdos con los diversos actores para avanzar (Núñez, 2003; Lemaitre, 1999: García-Huidobro y Cox, 1999, p.16).

La política de la reforma educativa (1996-2002) plantea como eje central la transformación curricular en un enfoque constructivista. Visión a la cual se suma una línea de refuerzo de la profesionalización docente; otra de implementación de la jornada escolar completa en los establecimientos escolares del país, y finalmente, la línea de reforma curricular, dirigida a construir y aplicar un nuevo marco curricular mínimo (García - Huidobro y Cox, 1999, p. 25). En los primeros años hubo acciones en líneas como el P-900 (Escuelas Focalizadas) y paralelamente se implementó en 1992 el Programa Mece Básica y se tramitó el Estatuto Docente (1991), promulgado finalmente en julio de 1991, más los Proyectos de Mejoramiento Educativo (PME). El bienio 19941995, significó la incorporación del macro Programa de Mejoramiento de la Calidad y Equidad de la Educación (MECE) para la enseñanza media, la ampliación del Proyecto Enlaces de informática educativa, todo con soporte financiero y técnico del Banco Mundial (García-Huidobro y Cox, 1999, p. 16; Hepp, 1999, p. 292).

Tales procesos se fueron implementado en paralelo con un incremento significativo de los recursos financieros del sector respecto del PIB, del gasto privado y del gasto público en educación (MINEDUC, 2002, p. 37). En materia de presupuesto, a partir del año 1995, educación se transformó en la prioridad del gobierno (MINEDUC, 2002), se incrementó su monto, se crearon nuevas subvenciones (siempre en el formato de subsidios) a la par de corregirse el Estatuto Docente (1996), se instaura el Plan de Desarrollo Educativo Comunal (PADEM) y el Sistema nacional de Evaluación del Desempeño (SNED) -entre varias iniciativas- que tenían por finalidad que al educación pública saliera de su tarea diaria en una visión de más largo plazo, y que existieran sistemas de incentivos para los establecimientos y sus docentes (González, 2003, p. 610). Complementariamente, los indicadores de eficiencia interna del sistema escolar mejoran, elevándose también los indicadores educativos, aunque con cambios menores en estos últimos (Bellei, 2003, p.149). Respecto de los docentes, se mejoran sus condiciones laborales y sus ingresos económicos, que hacia 1995 implicaron un 54,4\% de aumento promedio respecto del valor de 1990 (Rojas, 1998, p. 153).

Los resultados registrados entre los años 1995 y 2002 en los indicadores clásicos del sistema educativo se desplazaron positivamente. La educación avanzó lentamente, siendo más relevante el factor socioeconómico de

5 El concepto de política educativa agrupa múltiples expresiones orientadas al cambio, que en educación dan cuenta de una transformación significativa del sistema educativo o de parte relevante del mismo, atendiendo a su mejoría respecto de la situación inicial, que involucra la dimensión estructural, la histórica y la epistemológica (Popkewitz. 1994). 
la familia que la dependencia del establecimiento (si es público o privado), coincidiendo con el hecho que la sociedad chilena es la más desigual de la OCDE, y es una de las más desiguales del mundo (PNUD, 2003; Brunner y Elacqua, 2003). En consecuencia, el principal factor explicativo en los resultados escolares son los antecedentes socioeconómicos, variable que ha sido y sigue siendo dominante, al extremo que relega otras -como dependenciacon aportes marginales (Donoso y Hawes, 2002; Hsieh y Urquiola, 2002, 2006,Mizala y Torche, 2012).

La última fase de consolidación de las políticas (2003-2010), tiene características de estancamiento. Se inicia hacia fines del año 2002, tras aceptarse los resultados SIMCE y las comparaciones internacionales, el Ministerio propone 'llevar la reforma a la sala de clase'. "La respuesta de la política a este nuevo desafío está basada en dos pilares fundamentales: Primero una campaña para mejorar las habilidades de lectura, escritura y matemática de kinder a cuarto básico, lo que incluye el rediseño del curriculum de 1996 para los primeros cuatro cursos, cambiando las políticas de capacitación de los profesores, y haciendo esfuerzos especiales para obtener el apoyo de los padres. Segundo (...) el Ministerio de Educación se ha comprometido con el aseguramiento de calidad de los resultados aplicando (entre otras medidas) la evaluación docente y los requisitos de desempeño específicos" (OCDE, 2004, p. 35). Este fenómeno, pone a la vista la nueva dimensión de las tensiones.

La cobertura de la enseñanza básica se estabilizó desde el 2003 en un 97\% de la población en edad escolar respectiva, y la de enseñanza media sube al 96\% en el 2008 (MINEDUC, 2010, p. 25). Por su parte, la deserción en básica y media se mantiene en el orden del 1,4\% y 4.5\% correspondientemente (MINEDUC, 2010, p. 94). Para la enseñanza básica, la tasa de reprobación sube el año 2000 del 2,9\% al 4,5\% en el 2008, punto que a su vez se recupera en la tasa de abandono escolar del 1,4 al 1,2\% para los mismos años (MINEDUC, 2010, p. 80). La situación en la enseñanza media es más negativa en esos años, la reprobación pasa del 6,5 al 8,1\% y el abandono del 4,1 al 5,2\% (MINEDUC, 2010, p. 84). Finalmente, la tasa de éxito oportuno y éxito total se redujo de manera manifiesta: Para la cohorte 1980 - 1990 la primera era de 37,6 y la segunda de 64,9, mientras que para la cohorte 1992 - 2002 son del 54\% en éxito oportuno y 83.5\% de éxito total y para la cohorte 1996 - 2008 los valores son 73,5\% éxito oportuno y 89,4\% éxito total (MINEDUC, 2010, p. 90). Las implicancias de estos resultados impactan en una reducción del mayor tiempo de inversión del 28,3 al 14,2\% (MINEDUC, 2010, p. 80). Sin embargo,

La retención del sistema escolar en el nivel de Enseñanza Media, disminuye casi en un 2\% en 2007 y casi un 1\% en 2008, lo que implica que no sólo ha disminuido la cantidad de estudiantes que finalizan el nivel, sino también el número de estudiantes que son evaluados al final de cada año escolar (MINEDUC, 2010, p. 91).

En este marco es importante relevar que el gasto público es y ha sido la principal fuente de financiamiento para la educación de la mayoría de la población escolar (representa aprox. el 18\% del gasto total del Estado el año 2009), y pese a la incorporación del sector privado en esta tarea, se mantiene como la fuente más relevante al respecto, en especial para la enseñanza primaria y secundaria. Por ende, los recursos públicos conforman la base financiera del sistema escolar chileno, lo que es una constante fuerte de tensión.

Pero lo que no se vislumbró entonces fue que el modelo y mecanismos de operación financiera eran 'instrumentos de política educativa' tanto y más determinantes que las orientaciones políticas de la reforma. Así, operar una reforma educativa con un sistema de financiamiento asentado en otros principios, tensionó y complejizó el diseño político. En esta etapa los criterios articuladores del conjunto de las políticas educacionales: programas integrales de intervención de cobertura universal para el mejoramiento de la calidad de los aprendizajes y programas compensatorios focalizados en las escuelas y liceos de menores recursos con bajos resultados de aprendizaje para el mejoramiento de la equidad (OCDE, 2004, pp. 20-21),

no fueron suficientes para zanjar las determinantes económicas del sistema educativo. De hecho el "conflicto por el estancamiento de los resultados de logro de la educación" es un detonante clave del movimiento pingüino del año 2004, como también lo es para que la Reforma educacional de la Concentración pierda fuerza ya en el 2008 y prácticamente desaparezca del discurso público de los años siguientes, siendo las mismas autoridades sectoriales del Gobierno de entonces las que omiten hacer referencia a esta iniciativa -otrora incuestionable, 
atendiendo a la poca creencia que tenían sobre los resultados, no obstante se pudo el año 2010 y 2011 los resultados SIMCE y PISA evidencian que se experimentaron cambios relevantes.

La segunda hipótesis de trabajo plantea que las dificultades del sector educacional tienen su origen en la coexistencia de dos lógicas de operación en el campo, cuya dinámica se ha fundado en la incapacidad de dominio de una sobre la otra y que en la actualidad (2012) se vive una oportunidad histórica - producto de los acuerdos que han de adoptarse en los próximos años sobre la nueva institucionalidad de la educación (marco legal, financiero y técnico - educacional)- de equilibrar ambas visiones y proponer fundadamente orientaciones que permitan resolver esta antinomia en una propuesta de futuro que implique sentar las bases de una política de Estado en la materia.

Estas lógicas contrapuestas responden a concepciones del Estado alternativas, y por lo tanto al diseño de políticas y de procesos de implementación que generan conflictos y tensiones complejas de equilibrar, lo que se percibe en la forma como en el campo se han "articulado y resuelto" las políticas en este campo.

Ello que queda en evidencia en el informe de la OCDE sobre Chile (2004), donde se señala que más que cualquier otro país latinoamericano, Chile ha podido sostener el proceso de reforma debido al acuerdo político que prevalece. El cual incluyó

el esfuerzo por combinar dos políticas de reforma coexistentes pero ideacionalmente en conflicto. Una es la noción de que los mercados educacionales, con competencia entre escuelas, un alto grado de elección de los establecimientos educacionales por parte de los padres, y una administración privada de dichos establecimientos, proporcionan la mejor esperanza de eficiencia educacional y el rendimiento escolar más alto posible. La otra es la noción de que el gobierno central debe intervenir en el sistema educacional con una clara visión de lo que constituye una buen educación y cómo lograrla para asegurar que los estudiantes tengan la mayor oportunidad de aprender (OCDE, 2004, p. 289).

Lo relevante es que más allá del informe señalado, hay dos visiones dominantes que han entrado constantemente en conflicto, dando origen a una serie de arreglos -tensiones y equilibrios- y además, por sus implicancias sobre la subordinación de otras visiones que se han debido ordenarse tras estas dos corrientes (Inzunza, 2009: Caviedes, 2011).

La implementación de las políticas públicas en educación -en estos años- se ha movido entre conflictos y tensiones referidas a sus fundamentos, las acciones derivadas para su cumplimiento y las demandas de los diversos actores sociales que reclaman acceso a una educación de calidad impulsando equilibrios que no han resultado satisfactorios. Demanda que la educación pública ni la privada subvencionada han podido acoger debidamente (Consejo Asesor, 2006, p. 61) reflejando con ello la crisis del diseño político del Estado en educación (Redondo et al, 2007, Donoso, 2011). La cual reside en que las tensiones entre las políticas públicas y las demandas ciudadanas están en un punto crucial para el desarrollo del sistema educacional, por cuanto algunas de sus funciones políticas claves, a saber, fomento de la cohesión y de legitimación del orden social -en el sentido de Weber (1969) y Habermas (1992)- están comprometidas por los problemas que enfrenta la sociedad chilena: fuerte reproducción de la desigualdad intergeneracional y la incubación de nuevas formas de exclusión social que operan con formatos diferentes a los usualmente conocidos, originando fenómenos sociales emergentes (Larraín, 2010).

\section{Chile de las visiones en conflicto a la visión hegemónica del mercado}

La redemocratización de la sociedad chilena desde 1990 en adelante, no ha sido suficiente para redefinir nuevos pactos sociales plenamente incluyentes, tensionando la sociedad bajo dos lógicas de operación que se encuentran en un punto crítico. El conflicto latente se asienta en demandas sociales planteadas bajo nuevos códigos de desigualdad y exclusión social generados por la educación (Larraín, 2010), que considera elevados niveles formales (Ej.: educación media completa) pero que ocultan diferencias de calidad asociadas fuertemente al capital social de la familia (Consejo Asesor, 2006), situación que tensiona el hacer del Estado en educación y que da cuenta del conflicto entre los racionales en disputa. 
En este periodo hay distintas visiones sobre la finalidad de la educación y sus procesos de implementación, evidenciando una controversia significativa respecto del rol del Estado, que constituye el elemento clave de la política pública en educación: el derecho a la educación. Se instalan desde 1990 tensiones por un diagnóstico más optimista de la realidad, marcando el lento progreso en aquellas dimensiones educativas que estaban profundamente deterioradas (OCDE, 2004, p. 20). Aunque las autoridades del nuevo Gobierno sabían que no estaban respondiendo a las expectativas de los docentes, aceptaban los cambios a fin de mantener los equilibrios;

Tratar de reestructurar el sistema de financiamiento escolar en Chile produciría una fractura en el frágil equilibrio entre la izquierda y la derecha que formó parte implícita del acuerdo que reestableció el gobierno democrático (OCDE, 2004, p.106).

La reforma chilena que empezó como un proceso incremental, pasó a una fase de reforma propiamente tal centrada sobre ejes diversos. Algunas de las tensiones de la implementación de la reforma, identificadas por García-Huidobro y Cox, no pudieron ser resueltas, revelando la

Ausencia de un discurso unitario de la reforma que integre en un relato coherente y significativo el conjunto vasto de las distintas iniciativas y logre conectarlo con los temas de la tradición docente como: educación pública como función crucial del estado, la educación como actividad esencialmente moral -y secundariamente instrumental- rol central y de liderazgo del MINEDUC, solidaridad y no competencia, participación y no tecnocracia (1999, p. 44).

En sus orígenes estas visiones responden al ajuste social requerido para dar continuidad a la sociedad chilena en la década de los 90', y se mantienen vigentes en tanto no haya consenso para que impere una visión, implicando procesos constantes de pacto, que derivan tensiones y equilibrios para diseñar y -esencialmenteimplementar políticas que en su seno incluyan esta consideración.

En el actual escenario de la educación pública chilena (2012) las soluciones transitan por la lógica de distribución de los recursos regida por los criterios de mercado. Este último no considera las necesidades desde el punto de vista de lo que es justo (merecimientos por equidad), sino esencialmente desde la disponibilidad de recursos para adquirirles, fundando este considerando en que el mercado es particularmente eficiente al momento de asignar recursos, sin comprender las implicancias políticas y sociales de esta medida en el "pacto social vigente", salvo en el caso extremo de las población más vulnerable, a quienes se provee de estándares mínimos de calidad -en este caso- de educación.

Dos aspectos del diseño político en análisis resaltan: (i) la ausencia de criterios de "colaboración y cooperación" como factores operacionales en la gestión política y su reemplazo por la competencia; y (ii) la legitimación del "desfinanciamiento de la educación" de los que alcanzan tamaños o prácticas eficientes, frente a los que no lo hacen, siendo este aspecto un factor suficiente para definir y adoptar decisiones de continuidad de establecimientos y opciones educativas, desprovisto de los criterios de sentido y finalidad del servicio educativo. Esta materia amerita un análisis más detallado de las tensiones y equilibrios que depara.

Ambas dimensiones conforman claramente una de las mayores contradicciones no resueltas e incluso omitidas por el actual sistema de gestión y financiamiento de la educación imperante ${ }^{6}$ : una visión de corto plazo para una empresa que por su naturaleza es de largo plazo, situación que se vincula -precisamente- con los impactos del mercado: existe en las propuestas distributivas de recursos vía mercado una falta de comprensión y de proyección de la naturaleza del acto de educar, reducida tristemente al símil de la instrucción escolar de aprendizajes básicos, insuficiente desde cualquier punto de vista como sinónimo, y más como práctica pedagógica de una educación de calidad, esta discusión encierra una de las tensiones más poderosas que se han sustentado en estos años.

En estas condiciones, el país asume formalmente que la educación pública es implementada por todos los establecimientos escolares que reciben subvención financiera del Estado central, sean de propiedad privada (particular subvencionados) o sean municipales. Sin embargo, hay diferencias claras de derechos y deberes entre ambos tipos de propietarios, pese a que se busca minimizarlos mediante la LGE, los establecimientos públicos

6 Para muchos esto es la consecuencia evidente e insuperable de los fallos propios del mercado, que lo "inhabilitan" para cumplir con eficiencia esta tarea de distribución de los recursos con un horizonte de mediano y largo plazo. 
de enseñanza básica y media no pueden seleccionar estudiantes, deben atender a todo aquel que demanda matrícula, deben cumplir todas las regulaciones del Estatuto docente en materia de pagos y contrataciones y no pueden establecer sistemas de cobros adicionales (en enseñanza media con muchas restricciones) y deben hacer una rendición de cuentas bastante engorrosa en lo administrativo. Adicionalmente sus docentes son evaluados mediante un sistema nacional con implicancias para su desempeño laboral. Contrariamente, los establecimientos particular subvencionados, pese a recibir recursos públicos por la subvención estatal, pueden establecer cobros adicionales de mensualidades (financiamiento compartido), podían seleccionar estudiantes -algo que reduce la ley SEP hasta el $6^{\circ}$ grado- los docentes son regidos por el código del trabajo, con menores obligaciones financieras para los empleadores, sin que exista una carrera docente que regule promociones y salarios, sus sistemas contable son más livianos, y sus docentes no son evaluados bajo el procedimiento descrito. Las equivalencias en materia de gestión en este plano son escasas y resulta impropio definirles bajo el mismo concepto de educación pública, más aún cuando desde el tipo de población que atienden los establecimientos Municipales concentran la mayor cantidad de población vulnerable (31\% versus $10 \%$ de los particulares) y su vez a la población más vulnerable dentro de los vulnerables (Donoso y Arias, 2011).

El objetivo final de las políticas y reformas descritas es garantizar el doble principio del derecho a la educación de calidad y a la libertad de enseñanza, los que debiesen entenderse el uno en el otro recíprocamente. El sentido es que todos los establecimientos sostenidos con fondos públicos garanticen una educación de calidad y pleno acceso. Esto demuestra el grado inacabado del debate en aspectos sustanciales. A ello se suma las ambigüedades que las nuevas instancias de organización del sistema de educación contenidas en la Ley señalada y en las Proyectos de Fortalecimiento de la Educación Pública, como también las normativas referidas a la Superintendencia de Educación y la Agencia de Calidad, presentan aún muchas interrogantes, amén que su implementación ha sido bastante más lenta que lo esperado, creándose además problemas de gobernanza de magnitud en el sector.

El desenlace de este capítulo, con cierta seguridad será por el lado de incrementar las regulaciones y el establecimiento de mayores estándares, junto con medidas orientadas a mejorar la capacidad interna del sistema en sus diversos niveles, tanto para fiscalizar de manera eficaz las nuevas regulaciones y orientaciones, como para implementar dichos estándares.

En este escenario es importante poner en evidencia la raíz histórica de la desigualdad social en Chile y la desigualdad educativa, denunciados con evidencia empírica hacia fines de los años 50 (Ahumada, 1958; Hamuy, 1961) y sostenido con fuerza en los años 90 (Hsieh y Urquiola, 2002, 2006), visión sobre la cual existen algunos reparos pero que no invalidan el hecho de las marcadas desigualdades, sino por el contrario, son estos considerandos los que fundamentan la necesidad de un nuevo valor del subsidio según distintos grados de vulnerabilidad (Sapelli, 2006), más allá de la ley SEP, confirmando la vigencia de las fuertes diferencias sociales. Finalmente ello se trasunta en la fuerte estructura que muestran los resultados SIMCE en la serie de aplicación del año 2000 a la fecha.

Los diálogos inconclusos entre las concepciones de educación pública y algunas visiones sobre educación pública de gestión privada (con subvención estatal) se plasma en la Ley General de Educación, que no iguala ni derechos ni deberes entre ambos tipos de empresarios en los aspectos reseñados anteriormente, lo que demuestra la confusión que les invade, la cual además se expresa nuevamente en la primacía del "derecho a la libertad de enseñanza" por sobre el "derecho a una educación de calidad", como si ambos principios en los hechos fuesen contradictorios, no de cara al ideologismo que les sustenta, sino frente a las necesidades de los estudiantes. El conflicto entre ambos principios refleja la misma tensión de dos modelos políticos que han coexistido y que hoy el debate legislativo en torno a la institucionalidad del sector tiene una oportunidad y un desafío para equilibrar las dos lógicas en disputa, considerando que no ha existido consenso para que impere una sola, pese a los esfuerzos de la actual administración del Estado, expresados en el Panel de Expertos (2011), que contribuyen más a confundir sobre esta materia que a dar luces al respecto. 


\section{Referencias Bibliograficas}

AHUMADA, J. En Vez de la Miseria. Editorial del Pacífico, Santiago de Chile, 1958.

BELLEI, C.; GONZÁLEZ, P.; VALENZUELA, J. P. Fortalecer la Educación Pública un desafío de nivel nacional. En: Bellei, C., D. Contreras y J.P. Valenzuela Ecos de la Revolución Pingüina. Alcances, debates y silencios en la reforma educacional. Universidad de Chile, Santiago. pp.225 -256. 2010

BELLEI, C. Capítulo 3. ¿Ha tenido impacto la reforma educativa chilena? En: COX, C. (Editor). Políticas Educacionales en el Cambio de Siglo. La reforma del sistema escolar en Chile. Editorial Universitaria, Santiago. Pp. 125 -213. 2003.

BEYER, H.¿Qué hacer con la Educación Pública? Estudios Públicos, № 114, otoño. pp. 89 -126. 2009.

BEYER, H.; EYZAGUIRRE, B.; FONTAINE, L. La Reforma Educativa Chilena. En: Hevia, R. (Editor) La Educación ... Pp. 179 -202. 2003.

BOURDIEU, P. The Logic of Practice. Stanford University Press, Stanford, CA. 1990.

Distinction: A social critique of the judgment of taste. (R. Nice, Trans) Cambridge, MA: Harvard University Press. 1984.

BRASLAVSKY, C.; COSSE, G. Las actuales Reformas Educativas en América Latina: Cuatro Actores, Tres lógicas y Ocho tensiones. Preal/Documentos, Santiago, Chile. 1997.

BRUNNER, J.J; RLACQUA, G. Capital Humano en Chile, Universidad Adolfo Ibañez, Santiago. 2003.

CAVIERES, E. The Class and Cultural - Based exclusion of the Chilean Neoliberal Educational Reform. Educational Studies, 47, 111-132. 2011.

CEPAL-UNESCO. Educación y Conocimiento: Eje de la transformación productiva con equidad. Naciones Unidas, Santiago de Chile. 270 p. 1992.

CONSEJO ASESOR. Informe final del Consejo Asesor Presidencial para la Calidad de la Educación. Presidencia de la República, Santiago. 2006.

COX, C.; GONZÁLEZ, P. Políticas de Mejoramiento de calidad y equidad en la educación escolar en la década de los años 90'. En: COX, C., GONZÁLEZ, P. NúÑEZ, I., y F. Soto. 160 años de educación pública. Historia del Ministerio de Educación, Santiago. Pp. 101 -180. 1997.

COX, C. Capítulo I. Las políticas educacionales de Chile en las últimas dos décadas del siglo XX. En: C. COX (Editor). Políticas Educacionales en el Cambio de Siglo. La reforma del sistema escolar en Chile. Editorial Universitaria, Santiago. Pp. 19 -114. 2003.

DONOSO, S.; ARIAS, O. Diferencias de escala en los sistema de educación pública en Chile. Ensaio, no vol.19, n.71, $283-307$, abril/junho 2011.

DONOSO, S. La Reproducción de la desigualdad intergeneracional y nuevas formas de exclusión social producto del Diseño Político del Sistema Educacional Chileno. Cadernos CRH Salvador, Vol. 24, № 61, pp. 135 -152, jan/abr. Pp. 135 -152. 2011.

DONOSO, S.; SCHMAL, R. Introducción a la Economía de la Educación. El fenómeno educativo y sus connotación económica. Editorial Universidad de Talca, Talca. 2009.

DONOSO, S; HAWES, G. Eficiencia escolar y diferencias socioeconómicas: a propósito de los resultados de las pruebas de medición de la Calidad de la Educación en Chile. Educação e Pesquisa v. 28, № 2. Pp. 25-40. 2002.

FERNÁNDEZ, J. M. Manual de Políticas y Legislación Educativas. Editorial Síntesis Educación. Madrid 367 p. 1999.

GAJARDO, M. Reformas Educativas en América Latina: Balance de uma Década. Preal/Documentos, Santiago, Chile. 1999.

GARCÍA-HUIDOBRO, J.E.; COX, C. Capítulo I: La Reforma Educacional Chilena 1990 -1998. Visión de Conjunto. En: J.E. GARCÍA- HUIDOBRO (Editor). La Reforma Educacional Chilena. Editorial Popular, Madrid, 1999. Pp. 7 - 46. 1999. 
GARRETÓN, M.A.; GARRETÓN, R. La democracia incompleta en Chile: La realidadtras los rankings internacionales. Revista de Ciencia Politica, Vol. 30, No 1, 115 - 148. 2010.

GONZÁLEZ, P. Estructura Institucional, recursos y gestión en el sistema escolar chileno. En: COX, C. (Editor). Políticas ... Op. cit. Pp. 597 - 660. 2003.

HABERMAS, J. "The Limits of Neo-Historicism". Entrevista con J. M. Ferry. En J. Habermas, Autonomy and Solidarity. Londres. Verso. 1992.

HAMUY, E. El problema Educacional del pueblo de Chile. Editorial del Pacífico, Santiago de de Chile. 1961.

HAYEK, F.V. Los principios de un orden liberal. Estudios Públicos, № 2, 179-202. 1982.

HEPP, P. Capítulo 14. Enlaces: Todo un mundo para los niños y jóvenes de Chile. En: J.E. GARCíA-HUIDOBRO (Editor). La Reforma... Op. cit. Pp. 289 - 303. 1999.

HSEIH, C.; URQUIOLA, M. When school competes, how they compete? An assessment of Chile's nation wide school voucher program. World Bank's development group, Washington. 2002.

The effects of generalized school choice on achievement and stratification: Evidence from Chile's voucher program, Journal of Public Economics 90 1477- 1503. 2006.

INZUNZA, J. La Construcción del Derecho a la Educación y la Institucionalidad Educativa en Chile: Antecedentes para una Polémica de 200 años. OPECH, Santiago de Chile. 2009.

JOFRÉ, G. Subvenciones en Educación. Estudios Públicos, № 32, Pp. 31 -55. 1988.

LARRAÍN, J. Identidad chilena y el bicentenario. Estudios Públicos, Santiago de Chile, No 120, pp. 5-30. 2010.

LEMAITRE, M.J. Capítulo 6. El paso desde Mejoramiento a Reforma. En: J.E. GARCÍA-HUIDOBRO (Editor).

La Reforma... Op. cit. Pp. 129 -150. 1999.

MINEDUC. La Educación en Cifras. Ministerio de Educación, Santiago de Chile. 2010. . Indicadores de la Educación en Chile 2002. Ministerio de Educación, Santiago. 2002.

NúÑEZ, I. Pasado y futuro de la educación chilena. En: Hevia, R. (Editor) La Educación... Op. cit. Pp. 34 -44. 2003.

OCDE. Chile. Revisión de las políticas Nacionales de Educación. Organización para la Cooperación y el Desarrollo. París, 305 p. 2004.

Panel de Expertos para una Educación de Calidad. Informe Final Segunda Etapa. Fortalecimiento de la Institucionalidad Pública, Presidencia de la República, Chile, Mayo. 2011.

PNUD. Desarrollo Humano en Chile 2002. Programa de las Naciones Unidas para el Desarrollo. Santiago Chile. 2003.

POPKEWITZ, T. S. Sociología Política de las Reformas Educativas. Ediciones Morata, Fundación Paideia, Madrid.295 p. ver. Pp. 32-44. 2004.

REDONDO, J. et al. El derecho a la educación en Chile. Fundación Laboratorio de Políticas Públicas, Buenos Aires. 2007.

ROJAS, P. Remuneraciones de los Profesores en Chile. Estudios Públicos, № 71, Pp. 122-175. 1998.

SALAS, D. El Problema Nacional. Facultad de Filosofía y Educación. Universidad de Chile. Santiago. 1917.

SAPELLI, C. Desafíos del sistema educativo: La subvención preferencial. Temas de Agenda Pública, Año 1, №1. Pontificia Universidad Católica de Chile, Vicerrectoría de Comunicaciones y Asuntos Públicos, Santiago. 2006.

TEDESCO, J.C. Reformas Educativas en América Latina: Discusiones sobre Equidad, Mercado y Políticas Públicas. Serie Estudios Nº 14, Universidad de Talca, Instituto de Investigación y Desarrollo Educacional. Talca. 42 p. 1998.

VALENZUELA, J.P.; DE LOS RÍOS, D.; BELLEI, C. Evolución de la segregación socioeconómica de los estudiantes chilenos y su relación con el financiamiento compartido. Fondo Nacional de Investigaciones en Educación, Gobierno de Chile. 2008.

WEBER, M. La ética protestante y el espíritu del capitalismo, Fondo de Cultura Económica. 1969. 


\section{Algunos de los Hitos del Período}

1990 Reajuste de la subvención escolar.

1992 Inicio Programas MECE Básica, Enlaces, Educación Rural, P-900

Inicio de Investigaciones bases para la posterior Reforma

Conversación Nacional de la Educación Media.

1993 Inicio del Financiamiento Compartido

1994 Comisión Nacional de Modernización de la Educación

1995 Reajuste de la subvención escolar.

Inicio Programa MECE Media.

1996 Inicio de la Jornada Escolar Completa.

Comienzo de Programa de Pasantías en el Extranjero.

Decreto Supremo N 40 Marco Curricular Ed. Básica. Reforma Educacional

1997Inicio del Programa Montegrande.

Fin MECE Básica.

Inicio Programa de Formación Inicial Docente.

Publicación de los OFCMO.

1998 Decreto Supremo № 220 Marco Curricular Ed. Media y comienzo de la capacitación en la reforma curricular.

2000 Inicio Programa Liceo para Todos.

Fin MECE Media.

2002 Fin de la capacitación en la reforma curricular.

2003 Reforma constitucional de los 12 años de escolaridad, - Subvención pro retención.

Liceos para todos

2004 Fin Montegrande.

Promulgación de la Ley № 20158 de Evaluación del Desempeño Docente.

Requerimiento constitucional de la Alianza de Derecha contra la JEC.

2006 Inicio Programas de Escuelas y Liceos Prioritarios.

Fin de Escuelas Prioritarias.

Proyecto de Ley de Subvención Preferencial

Movimiento pingüino

Comisión Asesora presidencial para la Calidad de la Educación

2007 Proyecto de Ley General de Educación.

Aprobación de Ley de Subvención Preferencial.

2008 Proyecto de Ley de Sistema de Aseguramiento de la Calidad.

Proyecto de Ley que Fortalece la Educación Pública

2009 Aprobación de Ley General de Educación

Recebido em Outubro de 2012 Aprovado em Dezembro de 2012

7 Basado en Inzunza, J. (2009) op. Cit y complementado a partir de ello. 